\title{
La dimensión comunicacional en la emergencia de la innovación en el diseño turístico
}

The Communication Dimension in the Emergence of Innovation in Tourism Design

Arlette Bouzon

Université de Toulouse III arlette.bouzon@iut-tlse3.fr

Jesús Bermejo-Berros Universidad de Valladolid jbermejo@hmca.uva.es
Bouzon, A., y Bermejo-Berros, J. , (2015).

La dimensión comunicacional en la emergencia de la innovación en el diseño turístico.

Revista Internacional de Investigación en Comunicación aDResearch ESIC. No $12 \mathrm{Vol} 12$

Segundo semestre, julio-diciembre 2015. Págs. 28 a 45 DOI: 12.7263/ADRESIC.012.002 
El diseño constituye el lugar privilegiado de la emergencia de la innovación en los productos turísticos. La innovación es un factor clave para satisfacer a los clientes en mercados cada vez más globalizados y, al mismo tiempo, segmentados. En numerosos estudios anteriores se ha insistido en la influencia sobre la innovación de algunos factores como la introducción de las TICs. Sin embargo, hasta ahora, pocos trabajos han abordado el análisis del papel de la dimensión comunicacional en la emergencia de la innovación en el proceso de diseño turístico.

Tomando como antecedente una investigación de varios años, en varias empresas del sector turístico, en la que se ha utilizado una metodología de toma de decisión en situación (Naturalistic Decision Making), se presenta una reflexión teórica de cuyos

Clasificación JEL:

\section{M3}

\section{Key words:}

Innovación,

diseño,

turismo,

emergencia,

decisión en situación,

comunicación argumentos se desprende la necesidad de incluir, en los procesos innovadores de diseño turístico, la dimensión comunicacional. La primera parte del artículo se centra en la aparición de la innovación en el seno de las estructuras de proyecto e intenta aprehender el lugar que ocupa la comunicación en el proceso de diseño. La segunda parte se interesa por la toma de decisión en esos mismos procesos. La conclusión plantea que la toma en consideración de la dimensión comunicacional es garantía de éxito en el proceso de innovación en el campo turístico, dadas las complejas interacciones en el tiempo entre los agentes que intervienen en el diseño.

\section{ABSTRACT}

\section{JEL Classification: \\ M3}

\section{Palabras clave:}

Innovation, design, tourism, emergence, naturalistic decision making, communication
The design is the privileged place for the emergence of innovation in tourism products. Innovation is a key to satisfy customers who are in increasingly globalized markets and are, at the same time segmented. In many previous studies it has been emphasized the influence onto innovation of several factors, including the introduction of ICTs. However, until now, few works have addressed the analysis of the role of communication dimension in the emergence of innovation in the design process of tourism. Using as an antecedent an investigation conducted over several years, in which it has been used a method of Naturalistic Decision Making situation, we present a theoretical reflection of whose arguments it follows the need to include the communication dimension in the innovative processes of tourist design. The first part of the article focuses on the emergence of innovation within project structures and tries to capture the place of communication in the design process. The second part is concerned with decision making in those processes. The conclusion suggests that the consideration of the communication dimension is guarantee of success in the process of innovation in the tourism field, given the complex interactions over time between the actors involved in the design. 


\section{Introducción}

Las empresas turísticas, confrontadas a crisis diversas (económicas, ecológicas, políticas,..), buscan adaptarse, al tiempo que intentan controlar los riesgos asociados a esos cambios (seguridad, costos, plazos, ...). En ese proceso, la innovación constituye un recurso necesario, tanto para los consumidores deseosos de novedades, como para los inversores, siempre a la expectativa de oportunidades y de diferenciación comercial. Siendo un sector económico dinámico, está sujeto al influjo de factores que influyen sobre su grado de competitividad internacional. Es por ello que es necesario innovar para conservar parte del mercado mundial. Ahora bien, la innovación es multiforme y puede afectar tanto a un producto o un servicio como a la empresa que la propone (Peters, Pikkemaat, 2005).

La innovación ha sido objeto de numerosos estudios (Selmi, 2007, p. 256), consagrados a la empresa innovadora, a las controversias científicas o a la dirección de los proyectos industriales (Gramaccia, 2001; Bouzon, 2004 b, c). Pero son escasos los trabajos que se interesan por la emergencia de la innovación en la actividad de diseño llevada a cabo por los equipos de trabajo, particularmente en el campo de los productos o servicios turísticos. Ahora bien, éstos son cada vez más sofisticados y requieren la movilización de una pluralidad de saberes y actores, tanto hacia el interior como hacia el exterior de la empresa, que han de ser entendidos e integrados para poder alcanzar un objetivo de creación colectiva, controlando simultáneamente los riesgos asociados. Éstos pueden estar ligados a la disponibilidad de las instalaciones y a los medios de transporte, a la seguridad de los recorridos o a las dimensiones programáticas de los costes y plazos. A medida que el número de in- formaciones y conocimientos que han de ser movilizados aumenta, que los intercambios e interdependencias cognitivas son frecuentes y la estructuración de la actividad se hace difícil, e incluso imposible de precisar a priori (Terssac De, 1996), la cooperación de las personas implicadas en el proceso se convierte en un factor determinante (Palmberg, 2004). A ello se añade el hecho de que el diseño es también un proceso de toma de decisión que no siempre alcanza la unanimidad.

En el difícil contexto turístico actual, en permanente evolución, el diseño de los nuevos productos, concebidos a partir de las demandas y exigencias de los viajeros, lleva a hacer colaborar conjuntamente, de manera provisional, a individuos que unen sus competencias en función de su conocimiento respectivo de una región, de una lengua o de una cultura. Estas situaciones de concepción colectiva momentánea plantean entonces la difícil cuestión del alineamiento entre actividades (supuestamente generadoras de innovación al dominar la incertidumbre) e individuos, con competencias plurales, que disponen de una inevitable autonomía de acción. Pero este campo de investigación ha sido escasamente tratado. Los pocos trabajos existentes se focalizan principalmente sobre la actitud de los habitantes y de los turistas, sin estudiar las actividades llevadas a cabo por los diseñadores turísticos (tour-operadores, agencias de turismo, ...) y no permiten tener una visión de conjunto del concepto (Mbaye, 2014 ;Bessière, Poulain, Rayssac, 2012). Apenas abordan los fundamentos epistemológicos (Ethier, Morisser, Sarrasin, 2012) y no tratan algunas cuestiones importantes como la de conocer qué es lo que permite a los agentes implicados en el diseño trabajar juntos y participar activamente en una producción 
colectiva innovadora susceptible de satisfacer demandas exigentes.

Este trabajo tiene por objeto desarrollar un análisis reflexivo sobre esta cuestión recurriendo a las teorías disponibles y confrontándolas con los resultados de un estudio sobre el terreno, prolongado en el tiempo, que hemos llevado a cabo sobre los procesos de diseño de productos turísticos. A partir de una aproximación de investigación comprensiva, mediante observación sobre el terreno, en situación, desarrollada durante cinco años en el seno de cuatro empresas (un tour operator internacional, 2 PYMES y 2 agencias de viajes) del sector turístico francés, completada por un estudio cualitativo y una encuesta cuantitativa (Bouzon \& Devillard, 2011). Nuestra investigación busca la comprensión de los procesos de decisión a partir del sentido expresado por cada uno de los intervinientes en la acción. Este sentido sobrepasa el motivo de la acción, tal y como es enunciado por un actor, siendo más una reconstrucción a partir de la miríada de informaciones identificadas por el investigador, tanto en los individuos como en los contextos en los que evolucionan, y que ellos contribuyen a instaurar (Bouzon $\&$ Devillard, 2006).

Analizaremos estos procesos en términos de las tomas de decisión que intervienen al final de un período de comunicaciones más o menos prolongado y que, fuente de irreversibilidad, marca el compromiso ulterior en el curso de la acción (Fauré \& Bouzon, 2010). Nos veremos así confrontados a las tomas de decisión a partir de las prácticas (decision-as-practice), intentando ir más allá de las focalizaciones que hasta ahora se han centrado en el contenido o en el contexto, interno o externo (Papadakis, Thanos, Barwise, 2010; Llewellyn, Hindmarsh, 2010). Algunos trabajos indican que la dimension afectiva participa en las tomas de decisión, en particular en situaciones como aquellas en las que no se tiene tiempo suficiente para desmenuzar pormenorizadamente las alternativas en la toma la decisión (Kim et al, 2010 ; Kim et al, 2011 ; Chuang, 2007). No obstante, teniendo en cuenta las elecciones metodológicas de nuestro itinerario científico de carácter exploratorio, no nos focalizaremos aquí sobre los factores afectivos, de indudable interés, y que serán objeto de un trabajo de análisis ulterior.

Este artículo se inscribe así en la línea de los recientes trabajos, aún escasos, consagrados a la 'toma de decisión en situación', denominada NDM 'Naturalistic Decision Making', focalizados sobre los equipos de individuos que trabajan en contextos, muchas veces de urgencia, y observados en situación real. La metodología adoptada sigue de este modo un proceso de análisis de las decisiones en el momento en el que producen (Latour, 2005). Las decisiones, adoptadas en condiciones complejas, deben ser rápidas y eficaces, han de tomar en cuenta lógicas contradictorias y no jerarquizadas (Klein \& Klinger, 1991), un horizonte temporal limitado (Elliott, 2005 , p. 8), y alcanzar satisfactoriamente objetivos ambiguos, en evolución y/o múltiples (Klein, 1993; Zsambock \& Klein, 1997; Lipshitz et al., 2001; Kahneman \& Klein, 2009, p. 516). Como ejemplos de estas últimas cabe citar aquellas investigaciones que han analizado el control aéreo (Bisseret, 1981 ; Leplat \& Bisseret, 1965, Spérandio, 1978) o los sistemas industriales (Bainbridge, Beishon, Hemming \& Splaine, 1974 ; Bainbridge, 1978 ; Rasmussen, 1976). Estos trabajos se han ido ampliando progresivamente a un conjunto de situaciones que comparten características comunes tanto en Europa con, entre 
otros, los trabajos de Hoc et Amalberti (Hoc, 1993, 1996 ; Hoc \& Amalberti, 1994 ; Cellier, 1996), de Boreham (Boreham, Foster, \& Mawer, 1992) ou también de Moray (1986), como en Estados Unidos en el marco de la corriente denominada NDM (Naturalistic Decision Making) con trabajos como los de Klein, Orasanu, Calderwood \& Zsambok (1993) o Zsambok (1997), que critican abiertamente la incapacidad de las investigaciones consideradas clásicas para explicar los fenómenos observados en situación real de trabajo, fuera del laboratorio (Zsambok \& Klein, 1997).

Se han propuesto dos grandes formas de recogida de datos sobre la innovación (EUROSTAT y OCDE, 2006. p. 28), unas basadas en el sujeto y otras en el objeto. Las primeras se centran en las actitudes y las actividades de las empresas en su conjunto. En la recogida de datos utilizan prioritariamente métodos cuantitativos y técnicas de encuesta. Por su lado, las segundas, centradas en el objeto, tratan de innovaciones específicas y se emplean en el estudio de casos (Álvarez et al., 2008, p. 20). En nuestro caso, el estudio de las prácticas sobre el terreno reúne en una misma metodología datos procedentes de ambos enfoques, lo que le confiere valor ecológico que permite acercarse a la comprensión del papel del fenómeno comunicacional en el proceso de innovación. A partir de ello, la primera parte del presente trabajo se centra en la aparición de la innovación en el seno de las estructuras de proyecto e intenta aprehender el lugar que ocupa la comunicación en el proceso de diseño. A diferencia de numerosos estudios anteriores, que subrayan la importancia de otros factores, particularmente el papel de las TICs, las redes sociales (Vilaseca et al. 2007) u otros de naturaleza económica y social (Fagerberg \&
Mowery, 2005), en nuestro caso intentaremos mostrar la importancia de la dimensión comunicacional en el proceso crítico de innovación en el diseño. La segunda parte se interesa por la toma de decisión en esos mismos procesos. Tanto en una como en otra haremos referencia, a modo de ilustración, a extractos de entrevistas y situaciones sobre el terreno.

\section{El diseño de productos turísticos}

A partir del momento en que el producto a concebir alcanza una cierta amplitud o sobrepasa las fronteras nacionales, el diseño es generalmente conducido en el seno de un equipo de proyecto. Esta forma de trabajo busca sobre todo favorecer la transferencia de conocimientos y la puesta en común de las competencias. Organizada en torno a un responsable, el jefe de proyecto, esta estructura cooperativa entre actores permite, por una parte, sumar y poner en común los saberes, favoreciendo la 'creatividad colectiva' (Shapira, 1995) y, de otra parte, limitar la incertidumbre por el intercambio y la confrontación de los diversos puntos de vista de los actores implicados (receptores, hoteleros, animadores, ...).

El diseño, que abarca la actividad de elaboración de un producto previamente a su ejecución, puede ser innovador o rutinario según la originalidad de las características del producto turístico del que se trate o de los medios utilizados para su consecución (Selmi, 2007, p. 255). El diseño constituye de este modo uno de los espacios privilegiados de la empresa donde puede aparecer la innovación (Tseng, Kuo, 2007, p. 480), sabiendo que esta última emerge generalmente en las primeras fases del diseño, cuando el campo de posibilidades no está aún excesivamente restringido por las decisiones ya adoptadas. 


\subsection{Aparición de la innovación en el diseño de turismo}

La situación de los individuos no es idéntica durante el proceso de diseño: «algunos están involucrados en actividades de co-diseño, mientras que otros participan en actividades de diseño distribuido. Pueden encontrarse estas dos situaciones a lo largo de un mismo proceso de diseño y pueden asimismo ser tomadas en consideración sucesivamente por un mismo actor» (Darses, Falzon, 1996, p. 126).

En el co-diseño, las etapas del razonamiento son repartidas entre los participantes que desarrollan conjuntamente la solución. Comparten un idéntico objetivo a alcanzar y cada uno contribuye a su resolución colectiva con sus competencias específicas. En la concepción distribuida, en cambio, los actores que "están simultáneamente (pero no conjuntamente) comprometidos en el mismo proceso de cooperación, desempeñan tareas bien precisas, aquellas que han sido asignadas previamente, y persiguen por tanto objetivos (o cuando menos sub-objetivos) que les son propios, al tiempo que tienen también como objetivo participar, de la manera lo más eficaz posible, a la resolución colectiva del problema» (Darses \& Falzon, 1996, p. 127). Las partes respectivas de actividad de codiseño y de concepción distribuida ${ }^{1}$ difieren según los lugares y las personas implicadas en la actividad. Las interacciones entre los actores tienen así características diferentes dependiendo

1 Recordemos que el enfoque teórico del diseño distribuido se inscribe en el paradigma conexionista. La distribución, que está en el centro del modelo, estipula que los lenguajes y herramientas movilizados en cada uno de los campos de la actividad de diseño son diferentes. Apoyándose sobre el enfoque axiomático de Suh (1990), integra la noción de aparición o emergencia de un producto, fenómeno no predecible, aunque observable. Pero en este modelo, la comunicación entre los diversos individuos se hace únicamente por el envío de mensajes, lo que constituye una de sus limitaciones, y no la menos importante, (Bouzon \& Devillard, 2006) y que intentamos superar en el presente artículo. de que los individuos estén comprometidos en una situación de co-diseño o de diseño distribuido. Les guían dos objetivos complementarios, la sincronización cognitiva y la sincronización operativa.

Para los actores presentes en la situación, la sincronización cognitiva consiste en establecer un contexto de conocimientos compartidos, relativos a la situación y a la manera de afrontarlos. Este contexto concierne la demanda específica expresada por el viajero (sus limitaciones y sus deseos, más o menos claramente expresados) y las condiciones del viaje (vacunas y eventuales visados, duración, número de escalas, condiciones de alojamiento,...). Se trata «por una parte, de asegurarse que cada uno tiene conocimiento de los hechos relativos al estado de la situación: datos del problema, estado de la solución, hipótesis adoptadas y, de otra parte, asegurarse que los asociados comparten un mismo saber general en cuanto al campo: reglas técnicas, objetivos del campo y sus propiedades, procedimientos de resolución, etc.» (Darses, Falzon, 1996, p. 125). Esta sincronización cognitiva, dominante en situación de codiseño (en donde los actores del diseño participan conjuntamente en la resolución del problema), pero también presente en el diseño distribuido (cuando los individuos se coordinan al comienzo y al final de la tarea), se efectúa a través de procesos comunicativos en forma de explicaciones y justificaciones, modelización, construcción o mantenimiento de un «operativo referencial común» (Terssac de, Chabaud, 1990 ; Karsenty, Falzon, 1992).

En cuanto a la sincronización operativa, es dominante, incluso determinante, en el caso del diseño distribuido. Busca asegurar el reparto de las tareas a lo largo del tiempo entre los miembros del proyecto (puesta a disposición de un 
vehículo a la llegada de los viajeros, cálculo del tiempo necesario para llegar a un aeropuerto alejado, fecha de una operación festiva,...), y da lugar a actividades de coordinación, verbal o no. Con ocasión de esta sincronización operativa, los actores discuten y evalúan las consecuencias (y particularmente las limitaciones) que las eventuales soluciones, que pueden tomarse en consideración, implican en el trabajo de cada uno (prever un desvío para la visita de un lugar prestigioso, garantizar la compensación de un número de estrellas que no se corresponden con la demanda,...). Indudablemente, la ejecución se planifica pero cada individuo debe resolver individualmente su parte del problema. Con la excepción del jefe de proyecto, cuyas preocupaciones cubren el conjunto del producto, cada actor se interesa entonces, más particularmente, tanto por la definición de los interfaces de la parte que le concierne, como por las eventuales exigencias que las decisiones adoptadas por otros engendran sobre las suyas propias (modificación de los horarios de avión debido a un cambio de aeropuerto, toma en consideración del tiempo necesario para llevar a los viajeros por carretera, horarios de apertura de los lugares turísticos, ...).

Si la mayor parte de la actividad de diseño está distribuida entre los diferentes actores especializados (receptores, hoteleros, comerciales, servicio de visados, ...), se producen períodos de intensas interacciones, necesarios para alcanzar el resultado esperado, sobre todo durante las primeras fases del proyecto. Sin embargo, estas interacciones pueden ser relativamente restringidas y gestionadas en gran parte por el coordinador, quien se ocupa en ese caso de definir las interfaces. Pero esta gestión indirecta consume tiempo y también presenta ciertas dificultades debido tanto a la extensión de los análisis deta- llados a llevar a cabo como de la especificidad de las competencias a movilizar. Por lo tanto, la coordinación se convierte en una animación entre los actores que cooperan, lo que implica un cierto solapamiento de saberes y de lenguajes y un mínimo de implicación. En un proyecto complejo, como puede ser el caso de la visita de numerosos países de acogida o a ciudades distantes entre sí varios miles de kilómetros (China, América latina, Europa), el diseño está principalmente distribuido entre actores muy especializados que llevan a cabo, cada uno de ellos, tareas pertenecientes a su campo de competencia en una relación de «prescripción recíproca» (Hatchuel, 1996, p. 117). No obstante, esta relación está asentada sobre un contrato frecuentemente «implícito» (Kerbrat-Orecchioni, 1986; Hotier, 2001) que permite dar forma a los compromisos recíprocos habituales entre el mandatario (el especialista) y el encargado (jefe de proyecto). Según afirman los actores de las empresas que hemos estudiado, este tipo de contrato se utiliza hoy cada vez más en las empresas, haciéndose explícito en razón de la crisis que impone el establecimiento de asociaciones y diferencias culturales (por ejemplo, el arte de la negociación asiática bien diferente a la norteamericana). El pago sistemático de una señal tiene lugar desde la reserva, «lo que no era el caso años atrás, cuando funcionaba la confianza mutua». ${ }^{2}$

Las relaciones que se inscribían y se inscriben todavía en períodos de tiempo, se hacen hoy más efímeras y sometidas a los imperativos de productividad y rentabilidad. Siguen, sin embargo, el mismo tipo de evolución. Así, los actores implicados por una actividad de trabajo desarrollan a lo largo del tiempo relaciones in-

2 Entrevista del 3 de octubre de 2012 (Cf. Bouzon \& Devillard, 2011). 
terpersonales que se inscriben en lo que se ha convenido en llamar una «comunidad de prácticas» en un ciclo de vida previsible (Wenger, 1998). Al comienzo, los protagonistas del sector turístico, o no, no se conocen aún. Físicamente aislados (a menudo instalados en países diferentes), se conocen progresivamente, se reúnen, hacen intercambios y contribuyen a la creación de artefactos colectivos (documentos, informes, resúmenes, ...), a medida que avanza el trabajo. La aparición de ideas nuevas (por ejemplo, prever la visita de un pequeño parque temático de animales cuya próxima apertura sólo es conocida de los receptores locales; o también dar un rodeo para evitar un país en el que se ha producido recientemente un terremoto) y la confrontación de los puntos de vista (por ejemplo, renegociación de los costes suplementarios derivados de verse obligado a hacer un rodeo para evitar ese país) tienen lugar sobre todo en la fase preliminar, antes de que un proceso más formalizado conduzca a un diseño más detallado. Llegando a término, la intensidad de los intercambios en el proyecto disminuyen y la comunidad acaba desapareciendo, reapareciendo ocasionalmente más tarde para satisfacer una nueva demanda.

Para que el trabajo se efectúe y la innovación pueda eventualmente emerger, son necesarias algunas condiciones. Así, la innovación, que sigue caminos tortuosos, no marcados y a menudo a contra corriente (Alter, 2000), necesita un mínimo de inconformismo por parte de sus actores. Los diseñadores implicados necesitan un espacio de autonomía respecto a las reglas en vigor ${ }^{3}$. Una compartimentación de los individuos, una jerarquía demasiado opre-

3 Esta reflexión está presente sistemáticamente en las entrevistas con los agentes siva, una incitación al conformismo a las reglas del Management o a aquellas de los procedimientos establecidos, un ambiente de trabajo en el que la intolerancia y el «cada uno para sí mismo», conducen a la autocensura y a la retención de informaciones. Todo ello se revela como un freno a la innovación. De hecho, la coproducción innovadora implica un verdadero espíritu de confianza entre los individuos para que las ideas, aun mal explicitadas y afinadas, puedan ser enunciadas por algunos, sin temor al ridículo o a los juicios desfavorables y posteriormente enriquecidas por otros. «Las dificultades para transformar el conocimiento dentro de la empresa han sido observadas tanto en la teoría como en la práctica. Los mecanismos de transferencia del conocimiento a través de las fronteras y las culturas son difíciles» (Rolland Stanley, Perrin, 2007, p. 553). Este estado de gracia es frágil en un entorno que tiene tendencia en la actualidad a desarrollar la competencia individual. A esta situación se añade que hay cada vez más empleados contratados por cortos períodos y que no poseen necesariamente todas las competencias requeridas (becarios). La heterogeneidad del personal (formación, recorrido profesional,...) parece favorecer la apertura de espíritu, la tolerancia y la receptividad a las nuevas ideas, incluso si ello trae consigo también dificultades de comprensión y de comunicación (Bouzon, 2004 a). La calidad del ambiente de trabajo aparece así como una de las principales motivaciones de los actores interrogados en nuestras investigaciones sobre el terreno (Bouzon \& Devillard, 2011). Además, la innovación supone una aceptación de la toma de riesgos por parte de la organización y por tanto un cierto derecho al error concedido a sus autores. 


\subsection{Innovación y comunicación entre actores}

En estas estructuras de proyecto, donde el intercambio de información y la toma de decisión constituyen lo esencial de una actividad que siempre hay que recortar, sobre todo como consecuencia de las «exigencias de los viajeros siempre muy apresurados ${ }^{4} »$, las TICs se revelan indispensables (Selmi, 2007, p. 255) sobre todo con el denominado Yield managament (Selmi, 2007, p. 256). Estas tecnologías comienzan a contribuir de manera útil en la compensación de las bajadas en las comisiones de los profesionales del turismo que, en el futuro, podrán, por ejemplo, incluso plantearse la venta de tiempo de acceso a las informaciones turísticas, a poco que estas últimas sean completas y personalizadas según los perfiles y las características del turista potencial 5 .

Pero estas tecnologías generan igualmente restricciones pues eliminan, en particular, el cara a cara. Así, la larga difusión de informaciones se ve favorecida por la existencia de listas predefinidas que no engendran costes específicos para el emisor (ausencia de transmisión de papel) pero aumentan aquellos con cargo a los receptores (tiempo dedicado a la lectura e impresión múltiple). Las TICs acrecientan significativamente una «carga informacional» parcialmente útil (Bonneville, Grosjean, 2006, p. 36) y ofrecen una trazabilidad relativa de los intercambios, suponiendo el emisor, con razón o sin ella, que su correo será leído atentamente y archivado por su destinatario.

\footnotetext{
4 Según afirman la mayoría de los diseñadores que visiblemente lamentan esta presión.

5 La aparición de nuevos actores en la distribución en línea y su considerable aumento parece ir en ese sentido.
}

En las cuatro empresas estudiadas, los mensajes enviados precedentemente por nota de servicio, carta o fax, utilizan cada vez más este incierto canal: «Desde mi regreso de vacaciones, ya no tengo ni tiempo de leer mis correos... sobre todo porque muchos de ellos tratan de peticiones de información que cogen todo mi tiempo» ${ }^{6}$. Los actores se encuentran así, 'como copia adjunta', la mayor parte de la documentación del proyecto, sin ningún tipo de clasificación y selección previas. De esto modo tienen muchas dificultades para encontrar la información pertinente para las actividades propuestas. Redactados a menudo en un tono imperioso, los correos parecen imponer una respuesta inmediata, incluso un rápido acuerdo, integrando de hecho al individuo en una lógica de satisfacción inmediata. Este modo de intercambio es juzgado agotador por numerosos protagonistas en razón de la sobrecarga cognitiva que ello suscita. A ello se añade que, hoy día, parte de esa información circula por canales que añaden incertidumbre (por ejemplo, los SMS telefónico en los cuales los correctores ortográficos automáticos modifican los textos de manera inadecuada). Además, esos mensajes intercambiados, en la frontera entre lo escrito y lo oral (Hert, 1997), no siempre respetan las reglas elementales de la corrección y el civismo. Añadamos finalmente que, comentarios aparentemente anodinos, enviados a título informativo, son percibidos como requerimientos para llevar a cabo acciones que cultivan, por otro lado, la ambigüedad «No puedo garantizar que todos mis correos hayan sido efectivamente tomados en consideración. ¡Pero, si en algún momento aparece algún tipo de preocupación, cada uno sabrá sacar una copia en el buen momento para librarse!».

6 Los extractos de entrevista están indicados en itálicas. 
En estas organizaciones efímeras de intercambios mundializados, establecidos para una demanda precisa (un grupo de visajeros, un destino particular, una operación específica), los actores están, además, obligados a expresarse en inglés, lo que les supone indefectiblemente dificultades ligadas a la variabilidad de los sistemas lingüísticos. Así, el emisor nunca está totalmente seguro que su colega del otro lado del mundo descodificará con precisión el sentido de su mensaje (Morillon, Bouzon, Caria, 2006). Por otro lado, cada uno debe ordenar el abundante correo no solicitado que transita por Internet (Assadi \& Denis, 2005), cuyo origen, dudoso, es difícil de detectar por sus destinatarios en la masa de mensajes recibidos: «A pesar del antivirus, recibimos muchos correos de marketing directo, algunos de ellos sospechosos». Por tanto, cada uno de los protagonistas observados no parece fiarse sino de los colegas que conoce o con los que la empresa ha tratado ya.

Este entorno cotidiano es fuente de ambigüedad. Los conocimientos ya no son fruto de una iniciativa individual sino de una práctica colectiva, social, que se desarrolla gracias a la buena disposición de cada uno. En tal contexto, la confianza relativa (Laufer, Orillard, 2000), contingente, funciona a modo de coordinación entre los actores, evitando que cada uno tenga que verificar sistemáticamente la validez de las informaciones recibidas, o dominar saberes especializados cada vez más diversificados. Pero, en este contexto y en este universo jerarquizado de la empresa, ¿cómo se efectúa entonces el proceso de decisión en diseño?

\section{La decisión en situación}

Desde los trabajos pioneros de Herbert Simon, la toma de decisión en Management ha estado mo- delada bajo la forma de un proceso abstracto de resolución de problema (Simon, 1983), que articula tres fases (inteligencia, diseño, selección), permitiendo elaborar una solución racional procedente de un encadenamiento de distintas etapas. Sin embargo, este modelo canónico ha sido objeto de numerosas críticas que cuestionan la estructura linear del proceso de decisión en razón de los desvíos individuales y colectivos, del papel jugado por el contexto, de las intuiciones e, incluso, de las emociones, etc. (Cyert, March, 1963; Janis, 1972; Laroche, Weick 1995; Mintzberg, 1982). El reciente desarrollo de la corriente de «toma de decisión naturalista» (Lipshitz et al., 2001) da testimonio de la pertinencia de tales enfoques. En el abundante contexto de esta corriente de investigación, el estudio de las situaciones de diseño mientras se desarrollan (decision-as-practice), ofrece un campo privilegiado de estudio de la decisión, concebida como el resultado de la cooperación de diversos actores implicados en una actividad de trabajo, aunque estén a una distancia de miles de kilómetros. Entre varias personas, se ha de alcanzar un objetivo inaccesible para un actor individual en razón, sobre todo, del reparto de los saberes y de la inserción local de los actores implicados (receptores, hoteleros,...). Por otro lado, varias soluciones posibles pueden responder a la misma necesidad y ningún camino predeterminado conduce necesariamente a la solución que será finalmente adoptada. El resultado es a menudo incierto y el itinerario para llegar a él es poco conocido o, incluso, desconocido (Bouzon \& Devillard, 2006). Esta actividad depende especialmente de la importancia del producto (número de países visitados, cultura local, número de días de estancia, tipo de desplazamiento, características del alojamiento, número de viajeros 
implicados,...) y del carácter innovador de las propuestas adoptadas.

\subsection{Enfoque racional y acción situada}

La perspectiva cognitivista de la organización empresarial está fundada sobre la toma de decisión racional (decision-making). Esta aproximación está fundada sobre la distinción entre tomas de decisión y acción, incluso si «la responsabilidad compete a toda la organización, tanto la decisión como la acción, ya que estas dos fases son estrechamente solidarias» (Simon, 1983, p. 3).

Pero este enfoque racional no es aplicado sistemáticamente durante el proceso de diseño, que es también el resultado de una serie de «acciones situadas» que se apoyan en el contexto, sin aplicación de un plan preestablecido (Suchman, 1987; Weick, 1979, 1995) y haciendo intervenir asimismo interpretaciones narrativas de la situación por los agentes implicados (Bermejo, 2012). De hecho, la búsqueda y evaluación de soluciones alternativas se hace a menudo en la urgencia, en función de fuertes restricciones de coste y de plazos (entre los que destaca la fuerte demanda para el período estival). Asimismo, en caso de crisis (por ejemplo, virus del Ébola, guerra, huelga, seismo, ...), la primera solución juzgada a priori como satisfactoria, o que parece convenir a primera vista, es frecuentemente adoptada, sin ser objeto de análisis crítico en profundidad de todas las consecuencias y riesgos asociados (por ejemplo, cambio de ruta en el último momento). Además, la acción interviene en muchas ocasiones como respuesta a un contexto afectado por múltiples imprevistos, tales como una nueva demanda de un cliente, la no disponibilidad de un hotel previsto o del trayecto habitual de un viaje. Esta acción decisoria no se fundamenta, a menudo, sino en el mero «feeling » de los actores. Aquellas soluciones escogidas inicialmente, tras las modificaciones sucesivas, se encuentran desnaturalizadas, lo que las hace perder su atractivo (por ejemplo, no respetar las restricciones del turismo sostenible, en términos de emisión de gases a efecto invernadero, cuando al mismo tiempo la agencia se presenta como una empresa que practica un diseño turístico ecológico). Del mismo modo, el contexto puede contener objetos cognitivos que incorporan un cierto saber y orientan la acción. «Tras una demanda urgente de propuesta, se ha evocado una solución utilizada en un viaje anterior... siendo finalmente retenida! Pero sin análisis...». Adoptada como reacción a un contexto en movimiento, esta decisión es objeto eventualmente de una justificación a posteriori ante el cliente «...como habíamos reservado el hotel y habiamos pagado todo, hemos tenido que convencer al responsable del grupo que era la mejor solución... o al menos la única... a la vista de que no teníamos otra solución...».

La introducción de las TICs, ya evocada (Lotus Note, collaborative tolls, Mails, Databases) no se traduce forzosamente por una mejora de la eficacia en la organización y no contribuye sistemáticamente a un toma de decisión racional pues las herramientas propuestas no son compatibles con el funcionamiento y la cultura de la empresa implicada y, su implantación, es especialmente delicada. Estos métodos de trabajo en grupo proponen la gestión del conjunto de los intercambios comunicacionales en el interior del grupo de viajeros, destacando su componente colaborativo. Ahora bien, esta gestión presupuestaria centralizada modifica sensiblemente los procesos de decisión y el control de las actividades del conjunto del personal. Estas herramientas proponen de hecho nuevas prácticas, con una gestión común de las agendas indi- 
viduales y un seguimiento diario de las acciones de cada uno (número de citas semanales, tiempo consagrado a cada actividad, número de pedidos, montante de cada uno de ellos,...). Proponen también una difusión jerarquizada de la documentación y la puesta en común de los diferentes bancos de datos, cuando éstos existen (documentos de análisis, informes, resultados de las acciones, ficheros de clientes,...) «que facilitan la vida pero que están también ahí para controlarnos».

Por medio de las relaciones comunicacionales, estas herramientas pueden favorecer tanto la innovación turística de un equipo, al permitir el acceso de cada uno a la información, como frenarla al retirar a cada participante una parte de su autonomía o insertando ésta en un bloque de reglas de eficacia a corto plazo, poco compatibles con la búsqueda de innovación a más largo plazo. La calidad de la confrontación llevada a cabo durante los debates o a través de diversos documentos utilizados durante el proceso influye por añadidura en la decisión.

\subsection{Las condiciones de la confrontación de ideas}

Aunque la variedad de situaciones y su complejidad se acomodan poco a su generalización, la actividad de trabajo en el seno de las organizaciones turísticas estudiadas, aparece íntimamente ligada a la comunicación porque la cooperación de los actores implicados se ha hecho indispensable en razón del número elevado de informaciones y de conocimientos a manipular, de las interdependencias cognitivas y de la dificultad para estructurar a priori propuestas innovadoras de viajes a medida.

En el momento de la concepción de tales productos turísticos, todos los objetos contribuyen, de diferentes maneras, al trabajo a realizar (borrador, informe, previsiones, análisis, planning, bonos de viaje,...) y constituyen analizadores de éste. Estos «objetos intermediarios» permiten al investigador penetrar en el interior de la actividad de trabajo (Jeantet et al., 1996) y se revelan sobre todo como traductores (indicadores más o menos fieles) de lo que es ejecutado, puesto que marcan especialmente el estado de las relaciones entre los actores involucrados. Su carácter híbrido permite identificar la contribución de los individuos que participan en el proceso y que tienen acceso al contenido mismo de la actividad, así como aprehender su influencia, sobrepasando el mero valor operativo que les es atribuido. Denominados con diferentes acepciones ${ }^{7}$, son también artefactos cognitivos (Norman, 1993, p. $18)^{8}$, o soportes de memoria ${ }^{9}$, que permiten a los actores implicados tanto descargarles de ciertas tareas como estructurar sus actividades. Estos diversos objetos son igualmente mediadores que dan a cada uno de los colaboradores concernidos una representación común y efímera del proyecto en curso, lo que se considerada necesario a su propia actividad.

Ahora bien, la calidad de estos objetos es muy variable, sobre todo en el caso de las respuestas a las ofertas de empleo (por ejemplo, las ofertas públicas de empleo en hospitales o en ayunta-

7 Nombrados de diferentes formas por los investigadores (objetos, objetos-herramienta, objetos informativos o artefactos, disposición espacial, representaciones mediáticas u objetos intermediarios), todos estos diferentes soportes expresan la idea de una mediación entre la acción y el entorno.

8 Norman D., Les artefacts cognitifs. In Conein B., Dodier N., Thevenot L., (dir.) Les objets dans l'action. Raisons Pratiques, n4 , 1993. El objetivo del autor en este artículo es subrayar el papel que juegan los artefactos físicos en el procesamiento de la información, con la finalidad de integrarlos en la actual teoría de la cognición humana (p. 20).

9 Cf. el caso del « post it » 
mientos) y a veces los hace difíciles de analizar, sobre todo por razones de confidencialidad de los corresponsales extranjeros. Existen considerables diferencias respecto al afinamiento del análisis llevado a cabo en la elaboración de un nuevo producto turístico (por ejemplo, el «hecho a la medida de las masas» o aquel que afecta a un nuevo público objetivo como la tercera edad), y sobre todo respecto a la calidad y legibilidad de los documentos.

En las reuniones de trabajo, las circunstancias en las que se producen las confrontaciones de las diferentes propuestas no siempre son las óptimas. Así, las condiciones materiales de las reuniones, que permiten concluir una fase de concepción (acuerdo del cliente), permitiendo el arranque de la siguiente (reserva de plazas con pago de una señal), son a menudo difíciles. Los plazos de los que se dispone son cortos («siempre demasiado cortos» afirman los actores interrogados), a lo que se añade que los miembros del proyecto no pueden sustraerse de sus actividades habituales que deben siempre asumir en paralelo. La documentación (tipo de alojamiento, estado de las instalaciones, mapa de carreteras, ...) es voluminosa, dispersa, debe reconstituirse y analizarse en la urgencia, por un grupo formado por algunas personas. A ello se añade que esta documentación no siempre está disponible en su integridad y llega con retraso. Las informaciones mismas son imperfectas: en ocasiones poco legibles, mal estructuradas, escasamente sintetizadas, incluso incompletas. Estas imprecisiones y faltas de información (que constituyen el quid de la cuestión en los asuntos que se refieren a los equipamientos hoteleros, al impacto sobre el medio ambiente, al precio facturado por los servicios no incluidos en el paquete estándar, a las fechas y horarios de apertura,...) provoca situaciones de crisis (apertura del consulado, posibilidad de ser repatriado, relés de las compañías de seguros incompatibles con el circuito contratado) (Tseng, Kuo, 2007, p. 484). En ocasiones no existen alternativas y las justificaciones son imprecisas, lo que hace que las interrogantes planteadas por algunos clientes no puedan ser respondidas de forma inmediata. Estas reuniones con los proveedores se desarrollan en unos casos en inglés, lo que añade las dificultades ya evocadas anteriormente. Tanto el carisma individual como la reputación del actor implicado, o el hecho de que haya mantenido relaciones anteriormente (incluso efímeras), juegan un papel determinante en el tratamiento de las cuestiones tratadas. Por último, el debate está a veces sesgado por aspectos contractuales (con el subcontratista, los receptores,...), no debiendo descuidar los aspectos subyacentes. Del mismo modo, las respuestas formales limitan la amplitud de las innovaciones propuestas o intentadas por los diseñadores, lo que suscita ciertos grados de frustración.

\section{Conclusión}

El diseño constituye el lugar privilegiado de la emergencia de la innovación en los productos turísticos. Tiene lugar a partir de diferentes reflexiones individuales o colectivas conducidas por actores que movilizan sus conocimientos propios en una construcción social del saber y se coordinan progresivamente en base a una propuesta razonada, óptima, susceptible de satisfacer al viajero exigente. Pero la decisión en el seno de un grupo de proyecto de esta naturaleza es el resultado simultáneo de un procedimiento cognitivo racional, eventualmente sesgado, y de acciones situadas en contexto justificadas a posteriori. A la vista de las prácticas observadas, las 
teorías de perspectiva cognitivita y las de acción situada pueden de este modo ser útilmente movilizadas en un marco complementario y no contradictorio.

El análisis del proceso de diseño turístico en el que conviven actividades de co-diseño y de diseño distribuido muestra como se combinan, particularmente en los períodos de intensos intercambio, la coordinación entre actores que deben respetar las restricciones de coste, plazo, seguridad (Larson \& Gobeli, 1988) y los tiempos más largos de cooperación entre expertos (personal administrativo, comercial, técnico), cuya 'creatividad colectiva' (Shapira, 1995) es una de las condiciones de la toma de decisión relativa a la propuesta final. De este modo, este análisis permite aportar una respuesta a la interrogante inicial acerca de la intervención de los actores en el proceso de trabajo en el seno de un grupo de proyecto escindido en varios continentes. También permite comprender mejor como, por el recurso a las tecnologías de la información y la comunicación (TIC), cada uno de los actores participa activamente, con más o menos fortuna, en una producción colectiva innovadora susceptible de satisfacer las demandas renovadas de clientes cada vez más exigentes.

Finalmente, las condiciones de intercambio y de debate, oral o escrito, tal y como se ha señalado más arriba, adolecen de imperfecciones que pueden frenar la innovación. Para poder avanzar hacia una innovación eficaz en el diseño turístico es necesario tomar en consideración los diferentes aspectos que integran la dimensión comunicacional. «Aunque es fundadora (Barnard, 1938), omnipresente (Mintzberg, 1982) y constitutiva (Taylor, 1993)» (Giroux, 2006, p. 178), la dimensión comunicacional parece olvidada, incluso maltratada en este universo que sin embargo está bien informado de los beneficios de la «relación con el cliente». Ahora bien, estos diversos aspectos de la dimensión comunicacional, sobre los que hemos llamado la atención en este trabajo, internos a la propia estructura de trabajo, ocultados, pueden tener consecuencias externas perjudiciales, especialmente en términos de la imagen de los productos turísticos ofertados. Su estudio se hace, en consecuencia, imprescindible para entender y mejorar las dinámicas de innovación en la empresa turística.

El análisis en los niveles superiores de generalidad muestra que en las tomas de decisión del grupo de diseño turístico, intervienen tanto un proceso cognitivo racional, eventualmente distorsionado, como acciones situadas justificadas sólo a posteriori. A la vista de las prácticas observadas, las teorías cognitivistas y de la acción situada pueden (sin ser exclusivas) ser útiles, de manera complementaria y no contradictoria, para comprender los procesos de diseño turístico. 


\section{Bibliografía}

Alter N., (2000). Linnovation ordinaire. Paris : PUF.

Alvarez, A, Rego, G, Leira, J; Gomis, A., Caramés R, Andrade, M. (2008). Innovación turística: perspectivas teóricas y objetos de estudio. Rotur. Revista de ocio y turismo, no 1 - p. $19-50$ Assadi, H. and Denis, J. (2005). Les usages de l'email en entreprise : efficacité dans le travail ou surcharge informationnelle? En Kessous E., J.L. Metzger (Eds), Le travail avec les technologies de l'information. Paris: Hermès, p.135-154.

Avenier, M.J. (coord.) (1997). La stratégie «chemin faisant». Paris: Economica.

Bartunek J.M., Louis M.R. (1996). Insider/Outsider Team Research. Thousand Oaks: Sage.

Bationo-Tillon A., Folcher V., Rabardel P. (2010). Les instruments transitionnels : une proposition pour étudier la diachronie des activités narratives, Activités, vol. 7, n², p. 63-83.

Bermejo-Berros, J. (2012). The integration of the psychonarrative paradigm in the theory of Organizational Communication, Journal of Organisational Transformation \& Social Change, Vol. 9, 3, pp. 227-246

Bigley G., Roberts K. (2001). The Incident Command System: High Reliability Organizing for Complex and Volatile Task Environments, Academy of Management Journal, vol. 44, n 6, p. 1281-1299.

Biggs S.F., Wild J.J. (1985). An Investigation of Auditor Judgment in Analytical Review, Accounting Review, $\mathrm{n}^{\circ}$ 60, p. 607-633.

Bonneville, L., Grosjean, S. (2006). L'«Homo-urgentus» dans les organisations : entre expression et confrontation de logiques d'urgence. En Figures de l'urgence et communication. Communication et organisation, n²9, juin, p. 23-47.

Bouzon A. (2000). Les représentations sociales dans l'entreprise : de la cohésion à la conformité. En Pratiques de communication organisationnelle, sous la direction de P. Delcambre. Rennes: PUR. pp. 279-293.

Bouzon A. (2004 a). La place de la communication dans les systèmes à risques. Paris: L'Harmattan.

Bouzon A. (2004 b). Le partage des savoirs lors de la conception des systèmes à risque. En Metzger J.-P., Le partage des savoirs. Logique, contraintes et crises. Paris: LHarmattan. pp. 147-156.
Bouzon, A. (2004 c). Communication organisationnelle et TIC dans une relation de service en profonde mutation, XIVème Conférence Internationale du RESER, TIC et relations de services dans une économie globalisée: Enjeux pour les entreprises européennes. Castres, 23-25 septiembre, volume 2, p. 401-414.

Bouzon, A. (2006). Etudier la communication organisationnelle: champs, concepts, perspectives. Paris: L'Harmattan. p. 159-189.

Bouzon A., Devillard J., (2006). From Risk Assessment in an Uncertain Environment to its Communication : Expertise in Question. In Guedes Suares C. \& Zio E., Safety and Reliability for Managing Risks. Estoril (Portugal): Taylor \& Francis. p. 1281-1288.

Bouzon A., Devillard J. (2011). The Management of Uncertainty in Tourism: Strategic Paradoxes and Communication. Tourismos An International Multidisciplinary Journal of Tourism, Vol. 6, No 1, April 2011, p. 53-68.

Brackenbury, M. (2004). Has Innovation Become a Routine Practice that Enables Companies to Stay ahead of Competition in the Travel Industry? Paris: OECD Publishing.

Büscher M., Urry J. (2009). «Mobile Methods and the Empirical», European Journal of Social Theory, vol. 12, n 1, February, p. 99-116.

Catino M., Patriotta G. (2009). "Managing Errors and Blame in Extreme Organizations: The Case of the Italian Force", 25th EGOS Colloquium, Barcelona, 2-4 July.

Chi, M.T.H. (2006). Two Approaches to the Study of Experts' Characteristics, The Cambridge Handbook of Expertise and Expert Performance. Cambridge: Ericsson K., Charness N.

Chuang S.C. (2007): "The effects of emotions on the purchase of tour commodities", Journal of Travel and Tourism Marketing, vol. 22 no. 1, pp. 1-13.

Cyert R. et March J., (1963). A Behavioral Theory of the Firm, Second edition (1992), Oxford: Blackwell.

Darses F., Falzon P., (1996). La conception collective : Une approche de l'ergonomie cognitive. In Terssac G. de, Friedberg E., Coopération et conception. Paris: LHammathan. p. 123-137.

Decelle, F.X. and Tassin, J.F. (2005). Tourisme et Innovation. Bilan et Perspectives. Paris: La Documentation Française. 
De Keyser V., Nyssen A.N. (2009). The Management of Temporal Constraints in Naturalistic Decision Making: The Case of Anesthesia, Linking Expertise and Naturalistic Decision Making, Salas E. \& Klein G. (Eds.), New York, Psychology Press, p. 171-188.

Elliott T. (2005). Expert Decision-making in Naturalistic Environments: A Summary of Research, Australian Government Department of Defense, DSTO Systems Scientific Laboratory, Edinburgh.

eMarketer. (2006). Travel Agencies Online. Retrieved July 7 , from http://www.emarketer.com/Reports/All/Travel world aug06.aspx

Eurostat y OCDE (2005). Manual de Oslo. Guía para la recogida e interpretación de datos sobre innovación, Tragsa.

Fagerberg J., Mowery, D. et al (eds.) (2005). The Oxford Handbook if Innovation. Oxford: Oxford University Press.

Fauré B., Bouzon A., (2010). French approaches to the performative properties of language at work: an overview, $M a-$ nagement Communication Quarterly, vol 24, n² 4, p. 620-626.

Flin R., Stewart K., Slaven G. (1996). Emergency Decision Making in the Offshore Oil and Gas Industry, Human Factors and Ergonomics Society, 38, p. 262-277.

Forrester Research. (2006). European Travel Has Just Only Taken Off. Retrieved June 20, from http://www.forrester. com/Research/Document/Excerpt/0,7211,38994,00.html

Gioia D.A., Chittipedi K. (1991). Sensemaking and Sensegiving in Strategic Change Situation, Strategic Management Journal, vol. 12, n 6, p. 433-448.

Giroux, N. (2006). Le «nouage» des savoirs en organisation, In Bouzon A., Etudier la communication organisationnelle: champs, concepts, perspectives. Paris, LHarmattan, p. 159-189.

Green, Robert T., Cunningham, William H., Cunningham, Isabella C. (1975). The Effectiveness of Standardized Global Advertising. Journal of Advertising. $n^{\circ} 4$

Hatchuel A., (1996). Coopération et conception collective. Variété et crises des rapports de prescription. En Terssac, G. de, Friedberg E. (coord.), Coopération et conception. Toulouse: Octares, p. 101-122.

Hert P., (1997). Quasi-oralité de l'écriture électronique et sentiment de communauté dans les débats scientifiques en ligne, Réseaux, n¹7, p. 32-48.

Hotier H., (sous la dir.) (2001). Non-verbal et organisation. Paris : Editions L'Harmattan.
Hutchins, E. (1995). Cognition in the Wild, Cambridge: MIT Press.

Janis I.L., (1982). Groupthink: Psychological Studies of Policy Decisions and Fiascoes. Boston: Houghton Mifflin

Jeantet A., Tiger H., Vink D., Tichkiewitch S., (1996). La coordination par les objets dans les équipes intégrées de conception de produit. In Terssac G. de, Friedberg E., Coopération et conception. Paris, p. 87-101.

Kahneman D. et Klein G. (2009). Conditions for Intuitive Expertise. A Failure to Disagree, American Psychologist, vol. 64, $\mathrm{n}^{\circ} 6$, p. 515-526.

Karsenty L., Falzon P., (1992). Cadre général pour l'étude des dialogues orientés-tâche. In Six F., Vaxevanoglou X., (coord.), Les aspects collectifs du travail. Toulouse : Octares.

Kerbrat-Orecchioni C. (1986), Limplicite. Paris : Armand Colin

Kilm H., Park K., Schwartz N. (2010). «Will trip really be exciting? The role of incidental emotions in product evaluation», Journal of Consumer Research, vol. 36 no. 6, pp. 983-991.

Kim Y.H., Kim M., Goh B.K. (2011). «An examination of food tourist's behavior: Using the modified theory of reasoned action», Tourism Management, vol. 32 no. 5, pp. 1159-1165.

Klein G. (1993). A Recognition Primed Decision (RPD) Model of Rapid Decision Making, Decision Making in Action, Klein G., Orasanu J., Calderwood R. et Zsambok C.E. (Eds.), Norwood N.J.: Ablex, p. 138-147.

Klein G. et Hoffman R.R. (1993). Seeing the Invisible: Perceptual/Cognitive Aspects of Expertise, Cognitive Science Foundations of Instruction, Rabinowitz M. (Ed.), Mahwah, NJ.: Lawrence Erlbaum Associates, p. 203-226.

Klein, G.A., Klinger, D.W. (1991). Naturalistic Decision Making. CSERIAC Gateway, II(1), 1-4.

Lahlou, S (1996). Representations and the social co-ordination of action. 3rd Int. Conference on Social Representations, Aix-en-Provence, 9/1996. E EDF/DER HN5196020.

Lahlou, S., Fayard, A.L. (1998). Waiting for the Paperless Office: Two Video Tools for Investigating the Paperfull Office of Today. EDF-DER HN5198020.

Laroche H., Karl E. Weick (1995) Sensemaking in Organizations, Sage, Thousand Oaks, Californie. Sociologie du travail, 2/96, p. 225-232. 
Larson E.W., Gobeli D.H., (1988). Organizing for product development projects. Journal of Product Innovation Management, 5, pp. 180-190(11)

Lauffer, R., Orillard, M. (2000). La Confiance en Question. Paris: L'harmattan.

Lave, J. (1988). Cognition in Practice. Cambridge (UK): Cambridge U.P.

Lave J. et Wenger E. (1991, 1999). Situated Learning. Legitimate Peripheral Participation. Cambridge: Cambridge University Press.

Leplat J., Terssac G. de, (1990). Les facteurs humains de la fiabilité dans les systèmes complexes. Marseille: Octares

Lipshitz R. (2009). Puzzle-seeking and Model-building on the Fire Ground: A Discussion of Karl Weick's Keynote Adress, Linking Expertise and Naturalistic Decision Making, Salas E. et Klein G. (Eds.). New York: Psychology Press, p. 337-345.

Lipshitz R., Klein G., Orasanu J., Salas E. (2001). "Focus Article: Taking Stock of Naturalistic Decision Making", Journal of Behavioral Decision Making, $\mathrm{n}^{\circ}$ 14, p. 331-352.

Llewellyn N., Hindmarsh J. (Eds.) (2010). Organisation, Interaction and Practice. Studies in Ethnomethodology and Conversation Analysis. Cambridge: Cambridge University Press.

Lorimor, E. S., Watson Dunn, S. (1967). Four Measures of Cross-Cultural Advertising Effectiveness. Journal of Advertising Research, $\mathrm{n}^{\circ} 7$.

McLure, Wasko M., Faraj S. (2000). "It is what one does": Why people participate and help others in electronic communities of practice. Strategic Information Systems, 9, p. 155173.

Marshall N. (2008). Cognitive and Practice-based Theories of Organizational Knowledge and Learning: Incompatible or Complementary?, Management Learning, vol. 39, $n^{\circ} 4$, p. 413-435.

Mintzberg H., (1982). Structure et dynamique des organisations. Paris : Les Editions d'Organisation

Miracle, Gordon E. (1968). International Advertising Principles and Strategy. MSU Business Topics. $n^{\circ} 16$.

Morillon L., Bouzon A., Caria M., (2008). Les acteurs de l'entreprise face à l'émergence des TIC : des pratiques plurielles. Chapitre de livre, sous la direction de Jeannine Gerbault, La langue du cyberespace: de la diversité aux normes. Paris: LHarmattan. p.161-172
Norman, D.A. (1991). Cognitive Artifacts. En J. M. Carroll (ed.). Designing Interaction; Psychology at the Human-Computer Interface. Cambridge: Cambridge University Press.

Norman D., (1993). Les artefacts cognitifs. En Conein B., Dodier N., Thevenot L., (dir.) Les objets dans l'action, Raisons Pratiques, $n^{\circ} 4$.

Palmberg, C. (2004). The Sources of Innovation - Looking beyond technological opportunities. Economics of Innovation and New Technology, 13, p. 183-197.

Papadakis, V., Thanos, I., \& Barwise, P. (2010). Research on Strategic Decisions: Taking Stock and Looking Ahead. En P. C. Nutt \& D. C. Wilson (Éd.), Handbook of Decision Making : 31-69. Chichester: John Wiley.

Peters, M. and Pikkemaat, B. (eds) (2005). Innovation in hospitality and tourism, special edition of the Journal of Quality Assurance in Hospitality and Tourism, Vol 6, p. 3-6.

PhoCusWright. (2005). Online Travelmarket Statistics. Retrieved September 9, from http://www.channel.phocuswright. com/user.php?op=loginscreen\&module=NS-User\&errmsg= Rolland, N., Stanley, T., Perrin, A. (2007). Mechanisms of Knowledge Transfer Across Bounderies and Cultures: The Case of a Global Travel Firm, in Tourism, Mobility and Technology, N.Y.: Frédéric Dimanche (Ed), CERAM.

Salas E. et Klein G. (Eds.) (2009). Linking Expertise and Naturalistic Decision Making. New York: Psychology Press.

Salas E., Rosen M.A. et Diaz Granados D. (2010). "Expertise-based Intuition and Decision Making in Organizations", Journal of Management, vol. 36, n 4, July, p. 941-973.

Selmi, N. (2007). Yield management, a technological innovation in services: impacts on hotels and customers. TTRA Europe 2007 Conference, Tourism, mobility and technology, p. 255-262

Shafir E., Tversky A., (1997). Penser dans l'incertain. Raisonner et choisir de façon non conséquentialiste. In Dupuy J.P., Livet P. (dir.) Les limites de la rationalité, T.1. Paris: La découverte. p.118-150.

Shapira P. (editor), (1995). The R\&D workers. Managing innovation in Britain, Germany, Japan and the United-States. London: Quorum Books.

Shattuck L.G. et Lewis Miller N. (2006). Extending Naturalistic Decision Making to Complex Organizations: A Dynamic Model of Situated Cognition, Organization Studies, vol. $27, n^{\circ} 7$, p. 989-1009. 
Simon H.A. (1976). From Substantive to Procedural Rationality, Method and Appraisal in Economics, Latsis S.J. (Ed.), London, Cambridge University Press, p. 129-148.

Simon, H.A., Newell, A. (1972). Human Problem Solving. Englewood Cliffs, New Jersey: Prentice-Hall.

Simon H.A., (1983). Administration et processus de décision. Paris : Economica.

Strauss A., (1992). La trame de la négociation. Sociologie Qualitative et Interactionnisme, Paris, Éd. L'Harmattan.

Suchman, L. (1983). Office Procedure as Practical Action: Models of Work and System Design, ACM Transactions on Office Information Systems, vol. 1, Nº4, 320-328.

Suchman, L. (1987). Plans and Situated Actions, New York, NY: Cambridge U.P.

Suchman L., (1987). Plans and situated actions : The problem of human-machine communication. New York: Cambridge University Press.

Suh N.P., (1990). Principles of design. New York : Oxford University Press.

Taylor J., Cooren F., Giroux N., Robichaud D., (1996). The Communicational Basis of Organization : Between the Conversation and the Text, Communication Theory, vol. 6, no. 1, p.38-52.

Terssac de G., Friedberg E. (coord.) (1996). Coopération et conception. Toulouse: Octares.

Terssac G. de, (1992), L'autonomie dans le travail. Paris : PUF. Terssac G. de, Chabaud C., (1990). Référentiel opératif commun et fiabilité. En Leplat J., Terssac G. de, Les facteurs humains de la fiabilité dans les systèmes complexes. Marseille : Octares, 1990, p. 111-141.
Terssac G. de. (1996). Le travail de conception : de quoi parle-t-on ? Terssac G., Friedberg E., Coopération et conception. Marseille: Octares, p. 1-22.

Tseng, C., Kuo, H. (2007). Configuration of Innovation and Performance in the Hotel Industry: Evidence from Taiwanese Hotels, in Tourism, Mobility and Technology, Frédéric Dimanche (Ed), CERAM.

Vilaseca, J., Torrent, J., Lladós, J. \& Garay, L. (2007). Tecnologías de la información y comunicación, innovación y actividad turística: hacia la empresa en red, Cuadernos de Turismo, no 19 , pp. $217-240$

Weick K.E. (2009). "Tool Retention and Fatalities in Wildland Fire Settings: Conceptualizing the Naturalistic". En Salas E., Klein G. (Eds.). Linking Expertise and Naturalistic Decision Making. New York: Psychology Press, p. 321-336.

Weick K.E., (1995), Sensemaking in Organizations. Sage Publications, Fondations for Organizational Science, Thousand Oaks, CA.

Weick K. E., (1979). The Social Psychology of Organizing. Addison Westley, Reading, Mass., (2e édition).

Wenger E., (1998). Communities of Practice: Learning, Meaning and Identity. Cambridge MA, Cambridge University Press.

World Tourism Organization. (2006). Tourism 2020 Vision. Retrieved July 10, 2006, from http://www.world-tourism. org/facts/wtb.html

Zsambok C.E. et Klein G. (1997). Naturalistic Decision Making. Mahwah NJ: Lawrence Erlbaum Associates. 